\title{
Characteristics and Treatment Patterns of Patients with Advanced Soft Tissue Sarcoma in Korea
}

Hyo Song Kim, MD, $\mathrm{PhD}^{1}$
Chung Mo Nam, $\mathrm{PhD}^{2}$
Suk-Yong Jang, MD, $\mathrm{PhD}^{3}$
Sun Kyu Choi, MS
Minkyung Han, $\mathrm{PhD}^{5}$
Seonmin Kim, MS
Maria Victoria Moneta, MSc
Sae Young Lee, MD, $\mathrm{PhD}^{7}$
Jae Min Cho, MS
Diego Novick, MD, $\mathrm{PhD}^{8}$
Sun Young Rha, MD, $\mathrm{PhD}^{1}$

*A list of author's affiliations appears at the end of the paper.

Correspondence: Diego Novick, MD, PhD Eli Lilly and Company, Lilly Research Centre, Erl Wood Manor, Sunninghill Road, Windlesham, Surrey GU20 6PH, UK Tel: 44-1276-483-832

Fax: 44-1276-483-192

E-mail: novick_diego@lilly.com

Co-correspondence: Sun Young Rha, MD, PhD Division of Medical Oncology, Department of Internal Medicine, Yonsei Cancer Center, Yonsei University College of Medicine, 50-1 Yonsei-ro, Seodaemun-gu, Seoul 03722, Korea Tel: 82-2-2228-8130

Fax: 82-2-2227-7810

E-mail: rha7655@yuhs.ac

Received August 22, 2018

Accepted February 15, 2019

Published Online February 18, 2019

*Hyo Song Kim and Chung Mo Nam contributed equally to this work.

\section{Purpose}

A soft tissue sarcoma (STS) is a rare type of cancer, accounting for $1 \%$ of adult solid cancers. The aim of the present study is to determine the incidence of localized and advanced STS in Korean patients, their treatment patterns, and the survival of patients by disease status.

\section{Materials and Methods}

The STS patient cohort was defined using National Health Insurance Service medical data from 2002 to 2015. Incidence, distribution, anatomical location of tumors, survival rates (Kaplan-Meyer survival function) and treatment patterns were analyzed by applying different algorithms to the STS cohort containing localized and advanced STS cases.

\section{Results}

A total of 7,813 patients were diagnosed with STS from 2007 to 2014, 4,307 were localized STS and 3,506 advanced STS cases. The total incidence of STS was 2.49 per 100,000 person-years: 1.37 per 100,000 person-years for localized STS and 1.12 per 100,000 personyears for advanced STS. The 5-year survival rate after diagnosis was $56.4 \%$ for all STS, $82.4 \%$ for localized, and $27.2 \%$ for advanced STS. Half of the advanced STS patients (49.98\%) received anthracycline-containing chemotherapy as initial treatment after diagnosis.

\section{Conclusion}

This study provides insights into localized and advanced STS epidemiology, treatment patterns and outcomes in Korea, which could be used as fundamental data in improving clinical outcomes of STS patients in the future.

\section{Key words}

Epidemiology, Korean population, Advanced soft tissue sarcoma, Treatment pattern, Modality 


\section{Introduction}

Soft tissue sarcoma (STS) refers to a rare cancer which accounts for $1 \%$ of all malignant tumors [1]. Risks for sarcoma are not well understood and its development can be divided into environmental exposures, genetic susceptibility, and an interaction between the two [2]. The annual incidence rate ranges from 1.8 to 5 cases per 100,000 persons worldwide $[1,3,4]$. Every year, new cases reach an average of 12,310 in the United States [5], 23,574 in the European Union [6], and 412 in Korea [7,8]. STS is a heterogeneous group of cancerous tumors, comprised of more than 50 histological subtypes that develop from soft tissues of the body (e.g., fat, muscles, nerve tissues, deep skin tissues, visceral non-epithelial tissues) [9] with distinct molecular subtypes [10].

The 5-year survival rate of STS patients is about $50 \%$, with high variability depending on factors such as stage, size, primary site, metastasis, and tissue type of tumors [4]. About $40 \%$ to $50 \%$ of STS patients experience metastasis [11]. In patients with localized STS, surgery is the standard treatment, and radiotherapy and chemotherapy can be applied before and after the surgery [3]. Chemotherapy and/or radiotherapy can be used as the first treatment for patients not amenable to curative treatment with surgery. However, treatment modalities for incurable patients are very limited. Doxorubicin and ifosfamide are frequently used as first line treatment, either as monotherapy or as combination therapy $[4,12]$. Radiotherapy is also broadly used to treat metastatic STS patients [4]. Individualized treatments for advanced STS patients according to a variety of histologic and molecular subtypes of this disease should be encouraged, and the choice of chemotherapy should be adapted to the site of the disease and subtype [13]. Little, however, is known about real-world treatment patterns for advanced STS cancer. A deeper understanding of currently used treatments is needed to develop of new therapeutic protocols and treatment objectives.

The objectives of this study are to describe the incidence of localized and advanced STS cases in Korea, to investigate the epidemiological and clinical characteristics of STS patients, to analyze treatment patterns, and to estimate the survival rate of STS patients according to treatment type.

\section{Materials and Methods}

\section{Data source}

Medical data from the National Health Insurance Service (NHIS) in Korea was used. In Korea, around $97 \%$ of citizens are either national health insurance subscribers or dependents, and the remaining 3\% are medical aid beneficiaries. The NHIS keeps the claim data of the entire Korean population, consisting of around 50 million people. The Big Data Steering Department was established in 2014 [14], which covers data from 2002 to 2015 and includes the NHIS. The data extracted for this research included patient information (age, sex), treatment records, main/sub-disease codes (primary/secondary disease) and mortality.

\section{STS patient cohort definition}

To define the STS cohort, three inclusion/ exclusion criteria were applied: (1) all individuals with a first STS diagnoses as main disease code or first sub-disease code from 2007 to 2014 who were 18 years of age or older at the time of diagnosis (STS Initial Cases) were included; (2) individuals with a cancer diagnosis code in the previous 5-year period (20022006) were excluded since they might have had multiple primary cancers or other primary cancers with metastases to soft tissue; and (3) patients who survived more than 1 year without any relevant surgery, radiotherapy and chemotherapy records after first STS diagnosis were excluded. Some 10,091 patients were initially coded but finally excluded because they might be suspected to have sarcoma but this was not confirmed by pathologic analysis or they might be prevalent cases who had been diagnosed and treated before the index period. The International Classification of Diseases, 10th revision (ICD-10) diagnosis codes used for STS diagnosis are listed in S1 Table. Patients with diagnostic codes C40 and C41 were not included in the STS patient cohort as these codes were used both for chondrosarcoma and osseocartilaginous sarcoma, and therefore it was not possible to distinguish chondrosarcoma cases using these codes.

Subgroups were defined using an algorithm based on treatment type (surgery, radiotherapy, chemotherapy), treatment period and survival. The STS cohort was classified into five subgroups: (1) patients who received only surgery (surgery, OP), (2) patients who received only radiotherapy (radiotherapy, RT), (3) patients who received surgery and pre/ postoperative treatment (Pre-OP/Post-OP), (4) patients who received only chemotherapy with or without radiotherapy (systemic treatment, CTx/CCRT), or (5) those who died within a year of diagnosis (clinical deterioration $<1$ year, CD) regardless of treatments. Regarding the clinical deterioration 


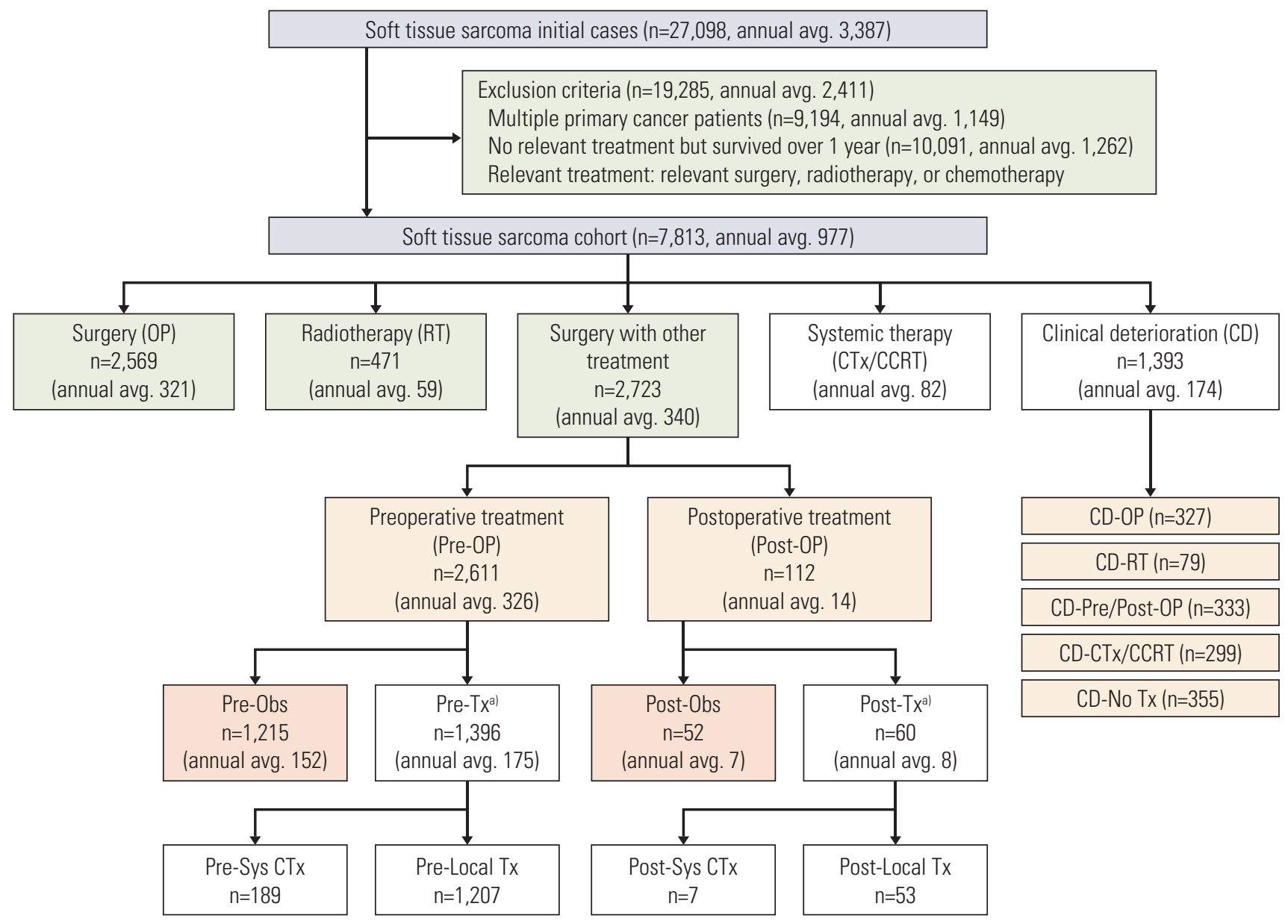

*White shaded boxes refer to advanced STS patients

Fig. 1. Taxonomy of soft tissue sarcoma (STS) patients by treatment patterns. Localized and advanced cases were defined using a diagnostic algorithm. OP, only surgery; RT, only radiotherapy; Pre/Post-OP, surgery in combination with pre / postoperative treatment; CTx / CCRT (systemic treatment), chemotherapy \pm radiotherapy; CD (clinical deterioration $<1$ year), patients who died within a year of diagnosis; Pre-Obs, patients who received surgery with preoperative treatment followed by observation; Pre-Tx, patients who received surgery with preoperative treatment followed by any treatment for cancer (chemotherapy, radiotherapy or surgery) 1 year after the initial surgery; Pre-Sys CTx, patients who received surgery with preoperative treatment followed by systemic chemotherapy 1 year after the initial surgery; Pre-Local Tx, patients who received surgery with preoperative treatment followed by local treatments 1 year after the initial surgery; Post-Obs, patients who received surgery with postoperative treatment followed by observation; Post-Tx, patients who received surgery with postoperative treatment followed by any treatment for cancer (chemotherapy, radiotherapy or surgery) 1 year after the initial surgery; Post-Sys CTx, patients who received surgery with postoperative treatment followed by systemic chemotherapy 1 year after the initial surgery; Post-Local Tx, patients who received surgery with postoperative treatment followed by local treatments 1 year after the initial surgery; $\mathrm{CD}-\mathrm{OP}$, patients who received only surgery and died within a year of diagnosis; CD-RT, patients who received only radiotherapy and died within a year of diagnosis; CD-Pre/Post-OP, patients who received surgery with pre/postoperative treatment and died within a year of diagnosis; CD-CTx/CCRT, patients who received systemic chemotherapy and / or radiotherapy and died within a year of diagnosis; CD-No Tx, patients who did not receive any treatment and died within a year of diagnosis. ${ }^{a} 1$ Year; the 1-year period was chosen because in clinical practice adjuvant therapy usually lasts about 1 year after surgery in Korea. 
Table 1. Baseline characteristics by subgroup

\begin{tabular}{|c|c|c|c|}
\hline Variable & $\begin{array}{l}\text { Total STS } \\
(n=7,813)\end{array}$ & $\begin{array}{l}\text { Curable STS } \\
(n=4,307)\end{array}$ & $\begin{array}{l}\text { Advanced STS } \\
\qquad(n=3,506)\end{array}$ \\
\hline Age (yr) & $54.5 \pm 16.2$ & $53.2 \pm 16.0$ & $56.1 \pm 16.4$ \\
\hline Median (min-max) & $55(18-101)$ & $53(18-97)$ & $57(18-101)$ \\
\hline \multicolumn{4}{|l|}{ Distribution by age (yr) } \\
\hline $18-29$ & $648(8.3)$ & $377(58.2)$ & $271(41.8)$ \\
\hline $30-39$ & $807(10.3)$ & $504(62.5)$ & $303(37.6)$ \\
\hline $40-49$ & $1,433(18.3)$ & $883(61.6)$ & $550(38.4)$ \\
\hline $50-59$ & $1,777(22.7)$ & $972(54.7)$ & $805(45.3)$ \\
\hline $60-69$ & $1,537(19.7)$ & $771(50.2)$ & $766(49.8)$ \\
\hline$\geq 70$ & $1,611(20.6)$ & $800(49.7)$ & $811(50.3)$ \\
\hline \multicolumn{4}{|l|}{ Sex } \\
\hline Male & $3,740(47.9)$ & $2,102(56.2)$ & $1,638(43.8)$ \\
\hline Female & $4,073(52.1)$ & $2,205(54.1)$ & $1,868(45.9)$ \\
\hline \multicolumn{4}{|l|}{ Initial diagnosis setting } \\
\hline General hospital & $7,354(94.1)$ & $4,060(55.2)$ & $3,294(44.8)$ \\
\hline Hospital & $249(3.2)$ & $132(53.0)$ & $117(47.0)$ \\
\hline Clinic & $209(2.7)$ & $115(55.0)$ & $94(45.0)$ \\
\hline Oriental clinic & $1(0.0)$ & 0 & $1(0.0)$ \\
\hline Comorbidities & $504(6.5)$ & $224(5.2)$ & $280(8.0)$ \\
\hline Cardiovascular disease & $24(0.3)$ & $10(0.2)$ & $14(0.4)$ \\
\hline Cerebrovascular disease & $35(0.5)$ & $18(0.4)$ & $17(0.5)$ \\
\hline Diabetes & $186(2.4)$ & $101(2.4)$ & $85(2.4)$ \\
\hline Liver disease & $142(1.8)$ & 57 (1.3) & $85(2.4)$ \\
\hline Others & $129(1.7)$ & $46(1.1)$ & $83(2.4)$ \\
\hline
\end{tabular}

Values are presented as mean \pm standard deviation or number (\%).

group, those patients were separately categorized because it was unclear whether mortality resulted from tumor progression or other causes. Pre-OP and Post-OP were subdivided into the patients who received surgery with pre/postoperative treatment followed by observation (Pre-Obs and PostObs) or who received surgery with pre/ postoperative treatment followed by any treatment for cancer (chemotherapy, radiotherapy or surgery) 1 year after the initial surgery (PreTx and Post-Tx). The 1-year period was chosen because in clinical practice adjuvant therapy usually lasts about 1 year after surgery in Korea. Pre-Tx and Post-Tx were subdivided into patients who received a local treatment (Pre-Local Tx and Post-Local Tx) and those who received systemic chemotherapy (Pre-Sys CTx and Post-Sys CTx).

Overall, we defined localized STS as follows: only local treatment (OP and RT), surgery combined with pre/ postoperative treatment followed by observation (Pre-Obs and PostObs). Advanced STS was defined as: only systemic treatment after diagnosis (CTx/CCRT) and rapid clinical deterioration within 1 year (CD), adding new treatment lasting longer ( $\geq 1$ year) than usual adjuvant treatment (Pre-Tx and PostTx).

\section{Statistical analysis}

Descriptive analyses were conducted on patient demographics and clinical characteristics/medical resources, as well as treatment patterns. STS incidence was calculated for 100,000 people using mid-year Korean Statistical Information Service [7] population data from 2007-2014. Anatomical locations of tumors based on ICD-10 codes were also analyzed. Comorbidities were defined as diseases for which a claim was made under the main or sub-disease code based on ICD10 codes related to the Charlson comorbidity index within three years prior to the STS diagnosis. Treatment patterns were analyzed based on the initial chemotherapy after diagnosis.

The survival curve was estimated using Kaplan-Meier product-limit estimator method; median survival time and 5 -year survival rate were calculated. The survival period of STS patients is the period from the date of the first diagnosis to the date of death. Patients were censored if they were still alive at the end of the follow-up period (December 31, 2015).

SAS ver. 9.2 (SAS Institute Inc., Cary, NC) was used for data management, statistical analysis, and plotting results. 


\section{Ethical statement}

The study was approved by the institutional review board at Yonsei University Graduate of Public Health (IRB Number: 2-1040939-AB-N-01-2016-421-01). The informed consent was waived as the NHIS database is anonymized in adherence to strict confidentiality guidelines.

\section{Results}

\section{Patient characteristics}

During the 8-year study period, from 2007 to 2014, the STS patient cohort was established with a total of 7,813 patients (Fig. 1). The demographic characteristics of localized and advanced STS patients at the time of initial diagnosis are shown in Table 1. The STS patients had an average initial diagnosis age (standard deviation) of $54.5 \pm 16.2$ years. The most populous age group was $50-59$ years (22.7\%), about half were females $(52.1 \%)$ and the initial diagnosis setting was a general hospital (94.1\%). The average age of the advanced STS patients was $56.1 \pm 16.4$ years, slightly higher than that of the localized STS patients ( $53.2 \pm 16.0$ years). The percentage of advanced STS in the $>60$ years age group was significantly higher than that in the 18-59 years age group $(\mathrm{p}<0.001)$. More than $90 \%$ of both localized and advanced STS patient groups were first diagnosed in a general hospital.

Only 504 out of 7,813 patients in the STS cohort had co-morbid conditions (6.5\%): 224 in the localized STS patient group $(5.2 \%)$ and 280 in the advanced STS patient group $(8.0 \%)$, based on the Charlson comorbidity index (Table 1). The most frequent co-morbidity among localized STS patients was diabetes $(2.4 \%)$. For advanced STS patients, the most common comorbidities were diabetes $(2.4 \%)$ and liver diseases $(2.4 \%)$.

\section{Incidence and distribution of STS patients}

There were 3,506 patients with advanced STS (44.9\%) and 4,307 patients with localized STS $(55.1 \%)$ in the STS cohort (Table 1). As sensitivity analyses, the numbers were recalculated using a 6- and 9-month period after the initial treatment as the time to define a case of advanced STS if a new treatment was received. The number of patients did not show a significant change, with $49.49 \%$ of patients at 6 months and $46.81 \%$ of patients at 9 months. Annual incidence cases of advanced STS decreased from 459 in 2009 to 397 in 2014, whereas that of localized STS increased steadily from 424 in 2009 to 743 in 2014, leading to the overall increase in annual

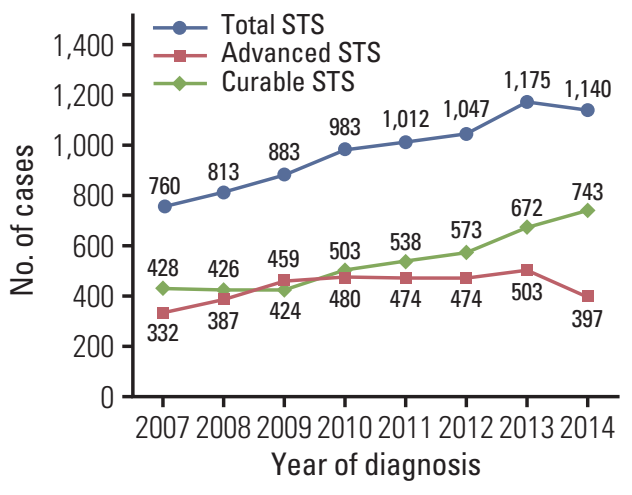

Fig. 2. Annual incidence cases by subgroup. STS, soft tissue sarcoma.

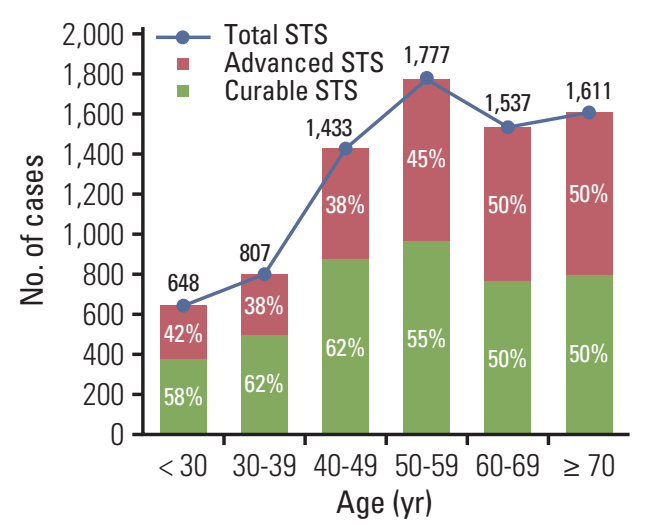

Fig. 3. Incidence cases by age and distribution by subgroup. STS, soft tissue sarcoma.

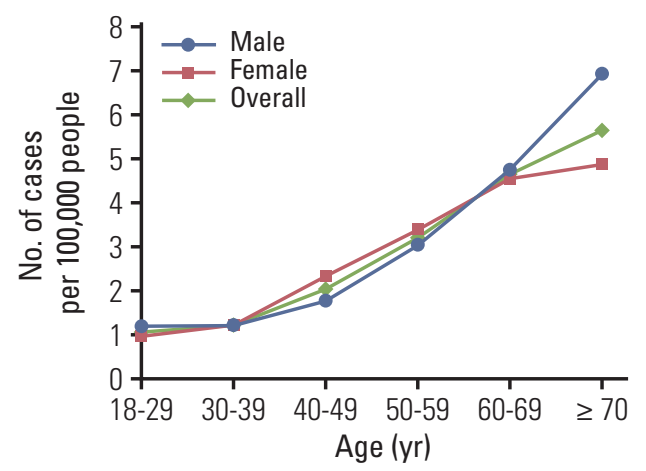

Fig. 4. Incidence rate by sex and age.

incidence cases of total STS (Fig. 2). Annual incidence cases by modalities are shown in S2 Table.

STS incidence was calculated for 100,000 people using mid- 
Table 2. STS incidence rate (per 100,000) by sex and age group

\begin{tabular}{|c|c|c|c|c|c|c|c|c|c|}
\hline \multirow{2}{*}{ Age group (yr) } & \multicolumn{3}{|c|}{ Total STS } & \multicolumn{3}{|c|}{ Curable STS } & \multicolumn{3}{|c|}{ Advanced STS } \\
\hline & Total & Male & Female & Total & Male & Female & Total & Male & Female \\
\hline $18-29$ & 1.07 & 1.16 & 0.96 & 0.62 & 0.65 & 0.59 & 0.45 & 0.51 & 0.38 \\
\hline $30-39$ & 1.22 & 1.21 & 1.23 & 0.76 & 0.73 & 0.79 & 0.46 & 0.47 & 0.44 \\
\hline $40-49$ & 2.06 & 1.78 & 2.35 & 1.27 & 1.12 & 1.43 & 0.79 & 0.66 & 0.93 \\
\hline $50-59$ & 3.22 & 3.04 & 3.40 & 1.76 & 1.65 & 1.87 & 1.46 & 1.39 & 1.52 \\
\hline $60-69$ & 4.65 & 4.75 & 4.56 & 2.33 & 2.51 & 2.17 & 2.32 & 2.24 & 2.39 \\
\hline$\geq 70$ & 5.64 & 6.94 & 4.86 & 2.80 & 3.71 & 2.25 & 2.84 & 3.22 & 2.61 \\
\hline Total & 2.49 & 2.41 & 2.57 & 1.37 & 1.36 & 1.39 & 1.12 & 1.06 & 1.18 \\
\hline
\end{tabular}

STS, soft tissue sarcoma.

Table 3. STS anatomical locations of tumor

\begin{tabular}{lccc} 
ICD-10 code & $\begin{array}{c}\text { Total STS } \\
(\mathbf{n = 7 , 8 1 3 )}\end{array}$ & $\begin{array}{c}\text { Curable STS } \\
(\mathbf{n}=4,307)\end{array}$ & $\begin{array}{c}\text { Advanced STS } \\
(\mathbf{n}=3,506)\end{array}$ \\
C49xx (other connective and soft tissues) & $5,255(67.3)$ & $3,199(74.3)$ & $2,056(58.6)$ \\
C48xx (retroperitoneum and peritoneum) & $1,910(24.5)$ & $806(18.7)$ & $1,104(31.5)$ \\
C47xx (peripheral nerves and autonomic nervous system) & $338(4.3)$ & $191(4.4)$ & $147(4.2)$ \\
C542 (myometrium) & $303(3.9)$ & $160(3.7)$ & $143(4.1)$ \\
C223 (hemangiosarcoma of liver, Kupffer cell sarcoma) & $91(1.2)$ & $13(0.3)$ & $78(2.2)$ \\
C224 (other liver sarcoma) & $54(0.7)$ & $10(0.2)$ & $44(1.3)$ \\
\hline
\end{tabular}

Values are presented as number (\%). STS, soft tissue sarcoma; ICD-10, International Classification of Diseases, 10th revision.

year Korean Statistical Information Service population data from 2007-2014. The total incidence of STS in the Korean population was 2.49 per 100,000 person-years (Table 2). A higher incidence was found in the "70 or older group" (5.64 per 100,000 person-years) and a lower incidence in the "18-29 group" (1.07 per 100,000 person-years). The incidence of localized STS was higher in all age groups, except in patients of 70 years or older. STS incidence increased with age. Furthermore, females showed a slightly higher incidence of total STS (females 2.57 per 100,000 person-years vs. males 2.41 per 100,000 person-years), localized STS (females 1.39 per 100,000 person-years vs. males 1.36 per 100,000 person-years) and advanced STS (females 1.18 per 100,000 person-years vs. males 1.06 per 100,000 person-years) groups.

If the incidence cases were analyzed by age and subgroup, the proportion of localized STS was higher than that of advanced STS in all age subgroups except the "60-69" and "70 or older" groups (Fig. 3). If the incidence rate of total STS was analyzed by sex and age, the incidence rate of males was higher than that of females in the "18-29," "60-69," and "70 or older" groups (Fig. 4). A similar trend was shown in localized STS. However, in advanced STS, the incidence rate of males was higher than that of females in the "18-29," "30-
39," and "70 or older" groups (Table 2).

\section{Anatomical locations of tumors in STS patients}

Anatomical locations of tumors based on ICD-10 codes were analyzed (Table 3). The main location for the total STS group was in "other connective and soft tissues" (C49xx, $67.3 \%)$ and the second main location was in "retroperitoneum and peritoneum" (C48xx, 24.5\%), while the remaining locations (C47xx, C223, C224, and C542) represented minor figures $(<10 \%)$. This distribution pattern was also observed in the localized STS group. However, the advanced STS group showed that tumors were located in different areas (C49xx, 58.6\%; C48xx, 31.5\%).

\section{Categorizing of STS patient cohort}

The STS patient cohort contained localized STS and advanced STS cases (shaded boxes in Fig. 1). The algorithm divided the STS patient cohort into five different, mutually exclusive groups, namely, patients with chemotherapy before or after surgery (Pre-OP or Post-OP group, 2,723 patients; 34.9\%), patients with only surgery (OP, 2,569 patients; $32.9 \%$ ), pati- 
ents who died within 1 year of diagnosis due to clinical deterioration ( $\mathrm{CD}, 1,393$ patients; $17.8 \%)$, patients who underwent chemotherapy only or chemotherapy with radiotherapy (systemic treatment group; CTx/CCRT, 657 patients; $8.4 \%$ ), and patients who underwent only radiotherapy (RT, 471 patients; $6.0 \%$ ).

Pre-OP or Post-OP patients (34.9\%) were further classified into the advanced STS group if they received new treatments (chemotherapy, radiotherapy or surgery) more than 1 year after the initial surgery. Patients who were still under care 1 year after the initial surgery but in which only clinical observation was provided were categorized as localized STS. PreOP or Post-OP patients were divided into patients who received chemotherapy before (Pre-OP, 2,611 patients) or after surgery (Post-OP, 112 patients). The number of patients who received surgery and postoperative treatment and went under observation without additional treatment was 52 (Post-Obs, 0.7\%). The number of patients who were just observed with no additional treatment after preoperative treatment and surgery was 1,215 (Pre-Obs, 15.6\%) and the number of patients who began a new treatment 1 year after surgery and postoperative treatment was 60 (Post-Tx, $0.8 \%$ ). Of these, seven patients (Post-Sys CTx, $0.1 \%$ ) were prescribed chemotherapy as a new treatment 1 year after surgery, and 53 patients (Post-Local Tx, 0.7\%) underwent local treatment (surgery/surgery and radiotherapy). A total of 1,396 patients received a new treatment 1 year after preoperative treatment and surgery (Pre-Tx, 17.9\%): in 189 of these, chemotherapy was prescribed more than 1 year after surgery (Pre-Sys CTx, $2.4 \%$ ), whereas 1,207 received a new local treatment (surgery / surgery and radiotherapy) (Pre-Local Tx, 15.5\%).

The $\mathrm{CD}$ group was defined as patients who died within 1 year of diagnosis due to rapid clinical deterioration $(C D$, 1,393 patients; $17.8 \%$ ). The CD group was divided into five subgroups according to treatment received: only surgery (CD-OP, 327 patients; 4.2\%), only radiotherapy (CD-RT, 79 patients; $1.0 \%$ ), pre/ postoperative treatment (CD-Pre/ PostOP, 333 patients; $4.2 \%$ ), systemic treatment (CD-CTx/CCRT, 299 patients; 3.8\%), and patients with no treatment (CD-No $\mathrm{Tx}, 355$ patients; $4.5 \%$ ).

\section{Survival rates}

The survival curve of each patient group was estimated using the Kaplan-Meier product-limit estimator method from diagnosis to death (Fig. 5). The 5-year survival rate after diagnosis was $56.4 \%$ in the total STS group, $82.5 \%$ in the localized STS group, and $27.2 \%$ in the advanced STS group. As expected, the survival rate of advanced STS patients was lower than that of localized STS patients. Median overall survival was 8 years in all patients but was only 1.76 years in the advanced STS group, much shorter than in the localized

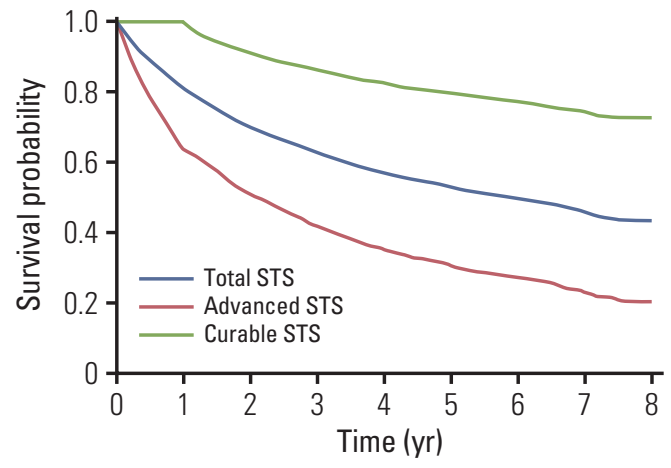

Total STS

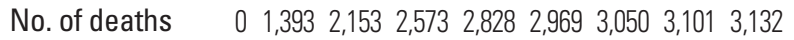

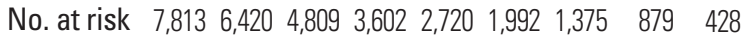

Advanced STS

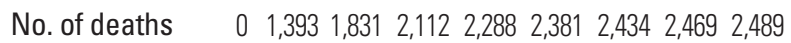

No. at risk $3,506 \quad 2,113 \quad 1,530 \quad 1,076 \quad 762 \quad 526 \quad 353 \quad 202 \quad 90$

Curable STS

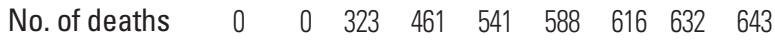

No. at risk 4,307 4,306 3,278 2,525 $1,957 \quad 1,465 \quad 1,021 \quad 676 \quad 338$

Fig. 5. Kaplan-Meier curve of overall survival. STS, soft tissue sarcoma.

STS group (more than 50\% of localized STS patients did not die during the follow-up period, and the median overall survival was not reached).

When the CD group was re-classified according to the treatment they received and then analyzed by treatment modalities, the 5-year survival rates after diagnosis by treatment were 74\% for OP+CD-OP, 70.1\% for RT+CD-RT, 53.1\% for Pre/Post-OP+CD-Pre/Post-OP, $28.6 \%$ for CTx/CCRT+ CD-CTx/CCRT and 0\% for CD-No Tx. Advanced STS cases from the 'Pre/Post-OP group showed a lower survival rate than the total Pre/Post-OP group and a higher rate than the CTx/CCRT group. Median overall survival was 5.73 years in 'Pre/ Post-OP+CD-Pre/ Post-OP' group, 1.85 years in 'CTx/ CCRT+CD-CTx / CCRT' group, and 0.24 years in 'CD-No Tx' group. Among the advanced STS, the median survival rate of the 'Pre/Post-Tx+CD-Pre/Post-OP' group was longer than that of the 'CTx/CCRT+CD-CTx/CCRT' group (3.43 years vs. 1.85 years) (Fig. 6).

\section{Analysis of chemotherapy patterns of advanced STS patients}

Only the initial chemotherapy after diagnosis of the advanced STS patients $(n=3,506)$ in the STS cohort was analyzed. In total, 2,299 of the advanced STS patients received at least one chemotherapy treatment after diagnosis $(65.6 \%$ of the 


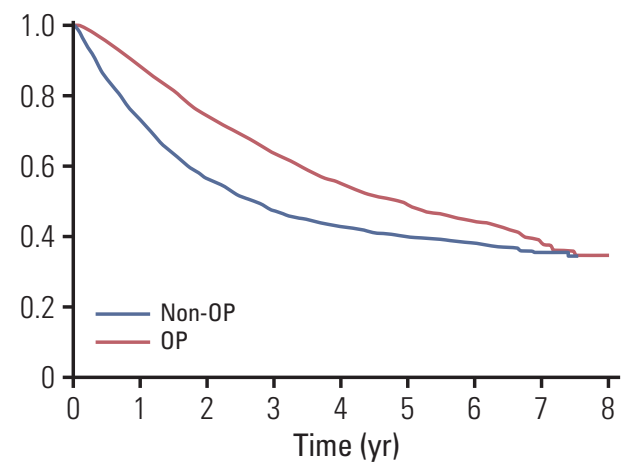

Non-OP (CTx_CCRT)

$\begin{array}{lrrrrrrrrr}\text { No. of deaths } & 0 & 299 & 494 & 575 & 607 & 621 & 627 & 632 & 633 \\ \text { No. at risk } & 956 & 656 & 392 & 250 & 180 & 129 & 90 & 55 & 28 \\ \text { OP (Pre-Tx+Post-Tx) } & & & & & & & & \\ \text { No. of deaths } & 0 & 333 & 577 & 777 & 921 & 1,000 & 1,046 & 1,076 & 1,095 \\ \text { No. at risk } & 1,789 & 1,456 & 1,137 & 825 & 581 & 396 & 262 & 146 & 62\end{array}$

Fig. 6. Kaplan-Meier curve of overall survival by treatment modalities in advanced soft tissue sarcoma (STS). The cases of $\mathrm{CD}$ (clinical deterioration $<1$ year) were included in the relevant groups according to the modalities. 'With surgery' $(\mathrm{n}=1,789)={ }^{\prime}$ Pre-Tx' $(\mathrm{n}=1,396)+{ }^{\prime}$ Post-Tx' $(\mathrm{n}=$ 60)+'CD-Pre/Post-OP' ( $\mathrm{n}=333$ ). 'Without surgery' $(\mathrm{n}=956)$ $={ }^{\prime} \mathrm{CTx} / \mathrm{CCRT}^{\prime}(\mathrm{n}=657)+{ }^{\prime} \mathrm{CD}-\mathrm{CTx} / \mathrm{CCRT}^{\prime}(\mathrm{n}=299)$. OP, only surgery; CTx/CCRT, chemotherapy \pm radiotherapy; Pre$\mathrm{Tx}$, patients who received surgery with preoperative treatment followed by any treatment for cancer (chemotherapy, radiotherapy, or surgery) 1 year after the initial surgery; Post-Tx, patients who received surgery with postoperative treatment followed by any treatment for cancer (chemotherapy, radiotherapy, or surgery) 1 year after the initial surgery; CTx/CCRT, patients who received only chemotherapy with or without radiotherapy.

STS advanced patient cohort); 657 were included in the CTx/CCRT group (28.6\%), 1,096 in the "Pre-Tx or Post-Tx" group (47.7\%), and 546 belonged to the CD group (23.8\%) (Table 4).

Of the advanced STS patients who underwent chemotherapy, 1,149 were treated with anthracycline $(50.0 \%)$. The largest fraction of patients used doxorubicin plus ifosfamide (458 patients, 19.9\%), followed by paclitaxel (385 patients, $16.8 \%$ ), others (372 patients, $16.2 \%$ ), ifosfamide (336 patients, $14.6 \%)$, gemcitabine and/or docetaxel (56 patients, 2.4\%), and pazopanib (1 patient, $0.04 \%$ ). When analyzing those patients who received anthracycline-containing chemotherapy by year, the prevalence of patients receiving anthracycline-containing chemotherapy in a neoadjuvant/adjuvant or metastatic setting was 229 in 2012, 245 in 2013, and 243 in
2014 (S3 Table).

Among the advanced STS patient subgroups, the chemotherapy patterns were slightly different. All patients in the CTx/CCRT group (657 patients) underwent chemotherapy after diagnosis, and the most frequently used regimen was paclitaxel $(20.4 \%)$, doxorubicin plus ifosfamide $(17.2 \%)$, and ifosfamide (13.4\%). Among the 1,456 patients in the Pre-Tx and Post-Tx groups, 1,096 patients $(75.3 \%)$ received chemotherapy at least once after diagnosis. In this group, doxorubicin plus ifosfamide was most frequently used $(22.3 \%)$, followed by ifosfamide (15.8\%), paclitaxel $(14.9 \%)$, and MAID (mesna, doxorubicin, ifosfamide, dacarbazine) regimen $(12.1 \%)$. In the CD group, the percentage of patients who received doxorubicin plus ifosfamide was $18.5 \%, 16.1 \%$ received paclitaxel, and $13.7 \%$ received ifosfamide. The Pre$\mathrm{Tx}$ and Post-Tx group had the largest proportion of patients who received anthracycline-containing chemotherapy (55.9\%), and the lowest number was in the CTx/CCRT group (42.0\%).

\section{Discussion}

This study, based on the analyses of the NHIS in Korea, found that about 977 patients are diagnosed with STS every year in Korea, $45 \%$ with advanced STS. The total incidence of STS has been estimated at 2.49 per 100,000 person-years, while it stands at 1.37 per 100,000 person-years for localized STS and 1.12 per 100,000 person-years for advanced STS. The 5-year survival rate was $82.4 \%$ for localized STS cases and $27.2 \%$ for advanced cases. Almost one out of five patients $(18 \%)$ died within 1 year of diagnosis. Most other patients (32.9\% of total) were treated solely with surgery. The firstline chemotherapy pattern of advanced STS patients after diagnosis was anthracycline-containing therapy in about $50 \%$ of the cases, followed by paclitaxel (16.8\%), ifosfamide $(14.6 \%)$, gemcitabine or docetaxel $(2.4 \%)$, and pazopanib $(0.04 \%)$.

The average age of patients with clinically advanced STS in our study was 56.1 years, and 53.2 years for localized STS patients. These figures are slightly lower than those reported by the Japanese registry (58.3) [15], the Surveillance, Epidemiology, and End Results report (SEER, 58 years) [16] and for a French cohort (61.5) [17]. Our study showed that the proportion of patients in the age category $20-40$ was $18.6 \%$; this was higher than in other solid tumors (lung, 1\%; liver cancer, $2 \%$; gastric cancer, $3 \%$; colorectal cancer, $3 \%$; and breast cancer, 10\%). Furthermore, the proportion of patients with comorbidities in this study (6.4\%) was lower than those reported by Kang et al. (12.9\%) [8] and Maretty-Nielsen et al. $(25 \%)$ [18]. This might be due to the nature of the data; while 
Table 4. Initial chemotherapy treatment for advanced STS patients

\begin{tabular}{|c|c|c|c|c|}
\hline Chemotherapy & Advanced STS & CTx/CCRT & $\begin{array}{c}\text { Post-Tx or } \\
\text { Pre-Tx }\end{array}$ & CD \\
\hline Total cohort & 3,506 & 657 & 1,456 & 1,393 \\
\hline Systemic therapy cohort & $2,299(100)$ & $657(100)$ & $1,096(100)$ & $546(100)$ \\
\hline Anthracycline-containing regimen & $1,149(50.0)$ & $276(42.0)$ & $613(55.9)$ & $260(47.6)$ \\
\hline Doxorubicin monotherapy & $249(10.8)$ & $60(9.1)$ & $127(11.6)$ & $62(11.4)$ \\
\hline Epirubicin monotherapy & $4(0.2)$ & $2(0.3)$ & $1(0.1)$ & $1(0.2)$ \\
\hline Doxorubicin+ifosfamide & $458(19.9)$ & $113(17.2)$ & $244(22.3)$ & $101(18.5)$ \\
\hline Epirubicin+ifosfamide & $21(0.9)$ & $6(0.9)$ & $8(0.7)$ & $7(1.3)$ \\
\hline CYVADIC regimen & $109(4.7)$ & $21(3.2)$ & $64(5.8)$ & $24(4.4)$ \\
\hline MAID regimen & $215(9.4)$ & $36(5.5)$ & $133(12.1)$ & $46(8.4)$ \\
\hline Other doxorubicin-containing & $93(4.1)$ & $38(5.8)$ & $36(3.3)$ & $19(3.5)$ \\
\hline Ifosfamide & $336(14.6)$ & $88(13.4)$ & $173(15.8)$ & $75(13.7)$ \\
\hline Paclitaxel & $385(16.8)$ & $134(20.4)$ & $163(14.9)$ & $88(16.1)$ \\
\hline Gemcitabine and/or docetaxel & $56(2.4)$ & $21(3.2)$ & $20(1.8)$ & $15(2.8)$ \\
\hline Pazopanib & $1(0.0)$ & 0 & 0 & $1(0.2)$ \\
\hline Others & $372(16.2)$ & $138(21.0)$ & $127(11.6)$ & $107(19.6)$ \\
\hline
\end{tabular}

Values are presented as number (\%). More than two were prescribed out: CYVADIC regimen, cyclophosphamide, vincristine, dacarbazine; MAID, In the case of prescribing one of (1) doxorubicin and dacarbazine, (2) ifosfamide and dacarbazine, or (3) doxorubicin and ifosfamide and dacarbazine. STS, soft tissue sarcoma; CTx/CCRT, patients who received only chemotherapy with or without radiotherapy; Post-Tx, patients who received surgery with preoperative treatment followed by any treatment for cancer (chemotherapy, radiotherapy or surgery); Pre-Tx, patients who received surgery with postoperative treatment followed by any treatment for cancer (chemotherapy, radiotherapy or surgery); Post-Sys CTx, Post-Tx patients who received systemic chemotherapy; Pre-Sys CTx, Pre-Tx patients who received systemic chemotherapy. ${ }^{a}$ Among Post-Sys CTx or PreSys CTx patients, frequency of medication used as first line: doxorubicin monotherapy 19 patients, doxorubicin and ifosfamide 45 patients, epirubicin and ifosfamide 1 patient, CYVADIC regimen 11 patients, MAID regimen 23 patients, other doxorubicin containing 9 patients, ifosfamide 24 patients, paclitaxel 34 patients, gemcitabine or doxetaxel 4 patients, others 26 patients.

our study is based on claim data, the Kang et al. [8] and Maretty-Nielsen et al.'s [18] studies were based on medical chart reviews of patients who underwent surgery for extremity STS and cancer registry analysis, respectively.

The total incidence of STS in our analyses of Korea (2.49 per 100,000 person-years) is within the reported incidences. The incidence is lower when compared with the incidence rates of some countries or regions, for example the United States (4.2 per 100,000 person-years) [19], Europe (4.7 per 100,000 person-years) [6], Ireland (4.48 per 100,000 personyears) [20] and the United Kingdom (4.51 per 100,000 person-years) [21]. However, the incidence is higher than the incidence rate reported for Sweden (1.8 per 100,000 personyears) [22] and Beijing (1.15 per 100,000 person-years) [23]. The incidence rates in our study are also higher than the estimated rate for Korea in the report by the International Association of Cancer Registries in five continents. The estimated incidence from 2003 to 2007 was 1.6 per 100,000 person-years in males and 1.2 per 100,000 person-years in females [24]. However, this last report only includes ICD-10 diagnosis C47 and C49, and they do not report C48, C223, C224, or C542 cases. As a matter of fact, some of the discrepancies in the incidence of STS across countries are due to the different diagnoses included in these studies. Bearing this in mind, it would be advisable to reach consensus on the establishment of consistent codes for STS to enable international comparisons. Our results showed that, after 2010, the incidence of localized STS increased more steeply than that of advanced STS. A possible explanation may be the early detection of cancer due to technological advances in imaging such as positron emission tomography and abdominopelvic computed tomography. Additionally, active workup of other diseases such as gastric cancer or colorectal cancer might have contributed to earlier detection of STS, even when asymptomatic.

Regarding anatomical location at the time of diagnosis, the advanced STS ratios at C47 and C49 were $43 \%$ and 39\%, respectively, while the advanced STS ratio at C48 was $58 \%$. C47 and C49 are associated with extremity STS, and early detection and treatment are relatively easy, whereas $\mathrm{C} 48$ is located 
in the peritoneum or retroperitoneum, which makes early detection difficult, and even if it is found, surgical treatment options are limited. Thus, clinical characteristics might explain the relatively higher ratio of advanced STS at C48.

The overall 5-year survival rate of the STS patient cohort after diagnosis was $56.4 \%$, which was similar to that reported by other countries: Ireland $(56 \%, 1994-2011)$ [20] and the United Kingdom (55\%, 2006-2010) [21]; close to the European average $(57.8 \%, 2000-2002)$ [6] but lower than in the United States $(65 \%, 2006-2012)$ [25] and Japan $(77.5 \%, 2006-2012)$ [15]. It should be noted that the Japanese study reported disease-specific survival rates. Considering its definition, in general, the disease-specific survival rate is higher than the overall survival rate. The overall 5 -year survival rate according to disease status was $82.5 \%$ in localized STS and $27.2 \%$ in advanced STS. This is comparable to the $83 \%$ for localized sarcoma and $16 \%$ for distant sarcoma reported in the United States, according to the SEER data [16]. The median overall survival rate of the total STS group was 8 years whereas it was 1.76 years for the group with advanced STS. This low median overall survival rate for advanced STS was similar to rates reported in previous studies (i.e., 1.17, Germany; 1.50, France, and 1.69, the United Kingdom) [17,26,27]. It suggests that advanced STS is a serious disease and that new treatment methods to improve the odds of survival are greatly needed.

The patterns of chemotherapy in advanced STS patients were also analyzed. Of those with advanced STS, a total of 2,299 patients $(65.6 \%)$ received chemotherapy. This was significantly higher than the $26.1 \%$ reported in Japanese studies [15]. Of the 2,299 patients who underwent chemotherapy, $41.6 \%$ (956 patients) received it in palliative settings, while in the Japanese study this was $28.9 \%$. The proportion of patients with metastatic disease at the time of diagnosis was similar in Korea (12.2\%) and Japan (11.4\%). The proportion of surgical treatment was $67.7 \%$ and $80.6 \%$, respectively. Most STS patients are treated by orthopedic surgeons in Japan and this clinical practice situation might contribute to the difference in treatment pattern.

Anthracycline-based chemotherapy is recommended as a standard-of-care in palliative settings in well-known guidelines such as the National Comprehensive Cancer Network (NCCN) and the European Society of Medical Oncology (ESMO). In this study, anthracycline-based chemotherapy was the most frequent treatment for advanced STS (50\%), which is consistent with previous studies from other countries such as Germany, the United Kingdom and Australia [26-28]. However, when analyzed by specific regimen, doxorubicin plus ifosfamide (AI) combination therapy was the most frequently used, whereas doxorubicin monotherapy was most frequently used in Germany, the United Kingdom, and Australia [26-28]. Pazopanib and gemcitabine +/- doc- etaxel use were only $0.04 \%$ and $2.44 \%$, respectively, which is much lower than in other countries. This might be because pazopanib received national reimbursement approval in July 2013 and gemcitabine + / - docetaxel was only used in some hospitals with pre-approval for off-label use of this regimen in Korea. Regimens in the advanced STS subgroup showed a higher percentage of AI, MAID, and CYVADIC regimen in the Pre/Post-Tx subgroup compared with the CTx/CCRT subgroup. Considering the fact that the Pre/Post-Tx group consists of patients who could receive surgical resection at the time of diagnosis, the clinical practice custom that combination therapies with a higher response rate were preferred may be reflected in this result. Compared with single-agent chemotherapy, combination chemotherapy seems to work well in terms of tumor response rate and progression-free survival but there seems to be no difference in overall survival in other clinical trials $[4,29]$. In the clinical trial that compared doxorubicin monotherapy with the combination of "doxorubicin plus olaratumab," the combined therapy demonstrated a statistically significant improvement in overall survival, resulting in a 50\% reduction of the hazard ratio [30].

Several limitations should be considered when evaluating these results. First, as our study is based on claim data, the limitation of all claim databases can be applied. Clinical data were limited because the primary purpose of claim data is reimbursement and not research. Consequently, localized/ advanced STS were distinguished based on the modalities patients actually received rather than disease-stage information. For example, we considered patients who received a new treatment for longer than 1 year as advanced STS cases. As most adjuvant chemotherapy and / or radiotherapy are completed within 1 year and the majority of recurrence occurred before 1.5 years [31,32], patients with the long treatment are likely to be the recurrent/relapsed cases. Further study is needed through linkage with the Korean Central Cancer Registry (KCCR) database that includes information on histology and stage. Second, when comparing our results with other studies, we should take into account that STS is a heterogeneous group of tumors and there is lack of consistency in classification and inclusion/exclusion criteria for each country. Finally, our study excluded patients with multiple primary cancer, which might have led to a possible underestimation of STS incidence.

To our knowledge, this is the first study conducted in Korea to investigate STS epidemiological and clinical characteristics and treatment patterns by analyzing the Korean NHIS Database that covers almost the entire Korean population. These results can be used as fundamental data to improve clinical outcomes in Korean STS patients since they not only provide information on current STS cancer incidence in Korea but also on actual clinical practices. 


\section{Electronic Supplementary Material}

Supplementary materials are available at Cancer Research and Treatment website (https:// www.e-crt.org).

\section{Conflicts of Interest}

Sae Young Lee, Jae Min Cho, and Diego Novick are employees of Eli Lilly and Company. All remaining authors have declared no conflicts of interest.

\section{Acknowledgments}

This study was supported by Eli Lilly and Company (project name: 2016-4276 Observational Study - Treatment Patterns, Resource Utilization and Characteristics in Patients with Advanced Soft Tissue Sarcoma in Korea: the National Health Insurance Service Database). Hyo Song Kim, Chung Mo Nam, Suk-Yong Jang, Sun Kyu
Choi, Minkyung Han, Seonmin Kim, Maria Victoria Moneta, Sae Young Lee, Jae Min Cho, Diego Novick, and Sun Young Rha performed the data collection, statistical analysis and manuscript writing. All authors read and approved the final manuscript.

\section{Author Details}

${ }^{1}$ Division of Medical Oncology, Department of Internal Medicine, Yonsei Cancer Center, Yonsei University College of Medicine, Seoul, ${ }^{2}$ Department of Preventive Medicine, Yonsei University College of Medicine, Seoul, ${ }^{3}$ Department of Preventive Medicine, Eulji University College of Medicine, Daejeon, ${ }^{4}$ Department of Biomedical Systems Informatics, Yonsei University College of Medicine, Seoul, ${ }^{5}$ Department of Public Health, Yonsei University Graduate School, Seoul, Korea, ${ }^{\top}$ Parc Sanitari Sant Joan de Déu, Fundació Sant Joan de Déu, CIBERSAM, Universitat de Barcelona, Barcelona, Spain, ${ }^{7}$ Eli Lilly and Company, Seoul, Korea, ${ }^{8}$ Eli Lilly and Company, Windlesham, UK

\section{References}

1. Wibmer C, Leithner A, Zielonke N, Sperl M, Windhager R. Increasing incidence rates of soft tissue sarcomas? A population-based epidemiologic study and literature review. Ann Oncol. 2010;21:1106-11.

2. Burningham Z, Hashibe M, Spector L, Schiffman JD. The epidemiology of sarcoma. Clin Sarcoma Res. 2012;2:14.

3. Casali PG, Blay JY; ESMO/CONTICANET/EUROBONET Consensus Panel of experts. Soft tissue sarcomas: ESMO Clinical Practice Guidelines for diagnosis, treatment and followup. Ann Oncol. 2010;21 Suppl 5:v198-203.

4. Schoffski P, Cornillie J, Wozniak A, Li H, Hompes D. Soft tissue sarcoma: an update on systemic treatment options for patients with advanced disease. Oncol Res Treat. 2014;37:35562.

5. Siegel RL, Miller KD, Jemal A. Cancer statistics, 2016. CA Cancer J Clin. 2016;66:7-30.

6. Stiller CA, Trama A, Serraino D, Rossi S, Navarro C, Chirlaque $\mathrm{MD}$, et al. Descriptive epidemiology of sarcomas in Europe: report from the RARECARE project. Eur J Cancer. 2013;49:68495.

7. Statistics Korea. Estimates of future population 2010-2060 [Internet]. Daejeon: Statistics Korea; 2011 [cited 2018 Dec 10]. Available from: http://kosis.kr./eng/.

8. Kang S, Kim HS, Choi ES, Han I. Incidence and treatment pattern of extremity soft tissue sarcoma in Korea, 2009-2011: a nationwide study based on the Health Insurance Review and Assessment Service database. Cancer Res Treat. 2015;47:57582.

9. Fletcher CD, Unni KK, Mertens F. World Health Organization classification of tumours pathology and genetics of tumours of soft tissue and bone. Lyon: IARC Press; 2002.

10. Corless CL, Schroeder A, Griffith D, Town A, McGreevey L, Harrell $\mathrm{P}$, et al. PDGFRA mutations in gastrointestinal stromal tumors: frequency, spectrum and in vitro sensitivity to imatinib. J Clin Oncol. 2005;23:5357-64.

11. Italiano A, Mathoulin-Pelissier S, Cesne AL, Terrier P, Bonvalot S, Collin F, et al. Trends in survival for patients with metastatic soft-tissue sarcoma. Cancer. 2011;117:1049-54.

12. Sheng JY, Movva S. Systemic therapy for advanced soft tissue sarcoma. Surg Clin North Am. 2016;96:1141-56.

13. Krikelis D, Judson I. Role of chemotherapy in the management of soft tissue sarcomas. Expert Rev Anticancer Ther. 2010;10: 249-60.

14. Seong SC, Kim YY, Khang YH, Heon Park J, Kang HJ, Lee H, et al. Data resource profile: the National Health Information Database of the National Health Insurance Service in South Korea. Int J Epidemiol. 2017;46:799-800.

15. Ogura K, Higashi T, Kawai A. Statistics of soft-tissue sarcoma in Japan: report from the Bone and Soft Tissue Tumor Registry in Japan. J Orthop Sci. 2017;22:755-64.

16. SEER cancer statistics review, 1975-2008 [Internet]. Bethesda, MD: National Cancer Institute; 2011 [cited 2018 Dec 10]. Available from: http: // seer.cancer.gov/csr/1975_20.

17. Ray-Coquard I, Collard O, Ducimetiere F, Laramas M, Mercier F, Ladarre N, et al. Treatment patterns and survival in an exhaustive French cohort of pazopanib-eligible patients with metastatic soft tissue sarcoma (STS). BMC Cancer. 2017;17:111.

18. Maretty-Nielsen K, Aggerholm-Pedersen N, Safwat A, Baerentzen S, Pedersen AB, Keller J. Prevalence and prognostic impact of comorbidity in soft tissue sarcoma: a population- 
based cohort study. Acta Oncol. 2014;53:1188-96.

19. Toro JR, Travis LB, Wu HJ, Zhu K, Fletcher CD, Devesa SS. Incidence patterns of soft tissue sarcomas, regardless of primary site, in the surveillance, epidemiology and end results program, 1978-2001: an analysis of 26,758 cases. Int J Cancer. 2006;119:2922-30.

20. Bhatt N, Deady S, Gillis A, Bertuzzi A, Fabre A, Heffernan E, et al. Epidemiological study of soft-tissue sarcomas in Ireland. Cancer Med. 2016;5:129-35.

21. Francis M, Dennis N, Charman J, Lawrence G, Grimer R. Bone and soft tissue sarcomas: UK incidence and survival: 1996 to 2010. London: National Cancer Intelligence Network; 2013.

22. Gustafson P. Soft tissue sarcoma. Epidemiology and prognosis in 508 patients. Acta Orthop Scand Suppl. 1994;259:1-31.

23. Yang L, Fang ZW, Fan ZF, Wang N, Yuan YN, Li HC, et al. An analysis of incidence trends and characteristics of soft tissue sarcoma in Beijing, 1999-2013. Zhonghua Zhong Liu Za Zhi. 2017;39:471-6.

24. Forman D, Bray F, Brewster DH, Gombe Mbalawa C, Kohler B, Pineros M, et al. Cancer incidence in five continents. Vol. X. Lyon: International Agency for Research on Cancer; 2014.

25. Surveillance, Epidemiology, and End Results Program [Internet]. Bethesda, MD: National Cancer Institute; 2016 [cited 2018 Dec 20]. Available from: http:/ / www.seer.cancer.gov.

26. Mytelka DS, Lorenzo M, Stafkey-Mailey D, D'Yachkova Y, Nagar SP, Candrilli SD, et al. Advanced soft tissue sarcoma: systemic treatment patterns and survival in Germany. Value
Health. 2016;19:A76.

27. Mytelka DS, Lorenzo M, Stafkey-Mailey D, D'Yachkova Y, Nagar SP, Candrilli SD, et al. Real-world treatment patterns and outcomes for patients with advanced soft tissue sarcoma receiving systemic therapy in the United Kingdom. Value Health. 2016;19:A751.

28. Bae S, Crowe P, Gowda R, Joubert W, Carey-Smith R, Stalley $\mathrm{P}$, et al. Patterns of care for patients with advanced soft tissue sarcoma: experience from Australian sarcoma services. Clin Sarcoma Res. 2016;6:11.

29. Judson I, Verweij J, Gelderblom H, Hartmann JT, Schoffski P, Blay JY, et al. Doxorubicin alone versus intensified doxorubicin plus ifosfamide for first-line treatment of advanced or metastatic soft-tissue sarcoma: a randomised controlled phase 3 trial. Lancet Oncol. 2014;15:415-23.

30. Tap WD, Jones RL, Van Tine BA, Chmielowski B, Elias AD, Adkins D, et al. Olaratumab and doxorubicin versus doxorubicin alone for treatment of soft-tissue sarcoma: an open-label phase $1 \mathrm{~b}$ and randomised phase 2 trial. Lancet. 2016;388:48897.

31. Hoang HL, Ensor K, Rosen G, Leon Pachter H, Raccuia JS. Prognostic factors and survival in patients treated surgically for recurrent metastatic uterine leiomyosarcoma. Int J Surg Oncol. 2014;2014:919323.

32. Potter DA, Glenn J, Kinsella T, Glatstein E, Lack EE, Restrepo $\mathrm{C}$, et al. Patterns of recurrence in patients with high-grade softtissue sarcomas. J Clin Oncol. 1985;3:353-66. 